\title{
Effect of frontal ablations upon an acquired temporal discrimination
}

MERLE E. MEYER, CAROL A. CFRAY, And CIIARLISS A. BLICK, Western Washington State College, Bellingham. Wash. $9 \$ 225$

Six food-deprived rats acyaired a temporal discrimination of a DRL 15 schedale and their performance on the same schedule were observed following frontal ablation. Postoperative deficit resulted from such ablations; however, that deficit varied considerably from $S$ to $S$ but in all cases was a transient deficit.

Since Jacobsen's writing in 1935, major interest has centered around the question of the neural structures involved with various aspects of timing behavior. Rosvold \& Szwarcbart (1964) have reemphasized the role of the frontocortical area and various associated subcortical structures and their interactions that are involved in temporal discrimination. delayed response performance, and general activity. From their model it is suggestive that bilateral lesions in the frontocortical area would disrupt the acquisition of temporal discriminations and that similar lesions would likewise disrupt the retention of an acquired temporal discrimination.

Meyer (1967) has shown that rats with marked frontal ablations or with caudate-putamen lesions learned various differential reinforcements of low-rate (DRL) schedules as effectively as sham controls. Similarly, Meyer and Colby ${ }^{1}$ have observed that bilateral lesions in the caudate-putamen impaired previously acquired DRL behavior, but that the animals' performance recovered with additional training. Recently, however, Schmaltz \& Isaacson (1968) report that animals with frontocortical lesions showed no post operative impairment when tested for retention and relearning of a DRL 20 reinforcement schedule. This present report is, in part. a replication of the Schmaltz and Isaacson study but where the frontal lesions were more extensive.

Subjects

The Ss were six hooded male rats approximately 130 days old at the start of the experiment. One animal died during surgery and a second animal did not reach the criterion during the preoperative phase and was dropped from the study.

Apparatus

The apparatus consisted of two operant chambers, each housed in a sound-deadened chamber equipped with white noise which served as an auditory mask. Standard relay equipment was used to program all reinforcement contingencies. The reinforcers were 45 -mg Noyes pellets and were dispensed under a DRL 15 contingency schedule. The responses and reinforcements were recorded on counters.

Preoperative Conditioning

All Ss were placed on a 22-h food deprivation schedule which continued throughout the study. One week later, the Ss were magazine trained, shaped, followed by two days under a continuous reinforement schedule with 100 reinforcements a day. The Ss were then directly shifted to the DRL 15 schedule and continued on that schedule until the $S$ reached the final eriterion performance of three successive 50-min daily sessions during which the $S$ received reinforcements on at least 50 , $i$ of the responses. After reaching the criterion the $S$ was subjected to bilateral ablation of the frontal corlex.

Surgical Procedures

All surgery was in a one-stage operation under generally clean conditions. The Ss were anesthetized with ether and held in a stereotaxic apparatus. Bilateral frontal ablations were produced by subpail aspiration of the desired portions of the brain. Following surgery all Ss were given intramuscular injections of penicillin.

Postoperative Conditioning

Seven days following surgery, the Ss were tested on the DRL 15 schedule without reshaping. The Ss continued on that schedule until they reached the final criterion performance of three successive 50 -min daily sessions in which the $S$ received reinforcements on at least $50^{\prime}$; of the responses.

Anatomical Findings

At the conclusion of the experiment, the Ss were sacrificed and perfused with $10 \%$ formalin, and the brain removed. Figure 1 illustrates approximations of the bilateral damage to the frontal cortex.

Behavioral Findings

All Ss regained criterion performance on the previously acquired DRL 15 schedule. However, there were wide differences in days required to regain their criterion performance levels. Figure 1 shows the days required to reach criterion level for each S. These data suggest that the retention and relearning of a temporal discrimination in a rat as measured by performance on a DRL schedule is affected by frontal cortex damage. However. the postoperative impaiment was transient and varied substantially among animals. These data do not clearly support the findings of Schmaltz and
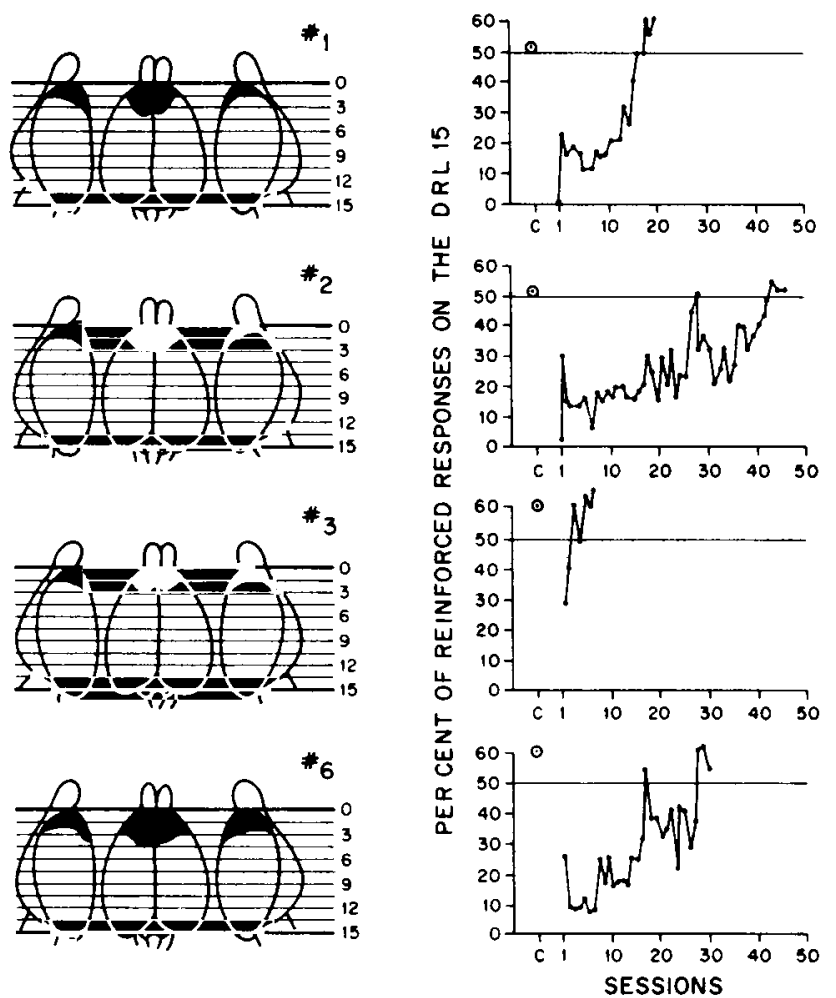

SESSIONS

Fig. 1. Reconstruction of frontal ablations and associated relearning of a DRL 15 schedule. The encircled dot at $C$ was the criterion level prior to the ablation. 
Isaacson in that they reported lesions of the frontal cortex had only slight affect on postoperative performance on a DRL schedule and they attributed the slight impairment to factors other than treatment. Nevertheless, they suggested that the extent of the frontal cortex damage could influence the postoperative deficit.

The findings of the present study show that retention and relearning of the DRL 15 schedule of reinforcement is influenced by frontal cortical ablation and that the effects are transient when the lesions to the frontal lobes are extensive.
REFERENCES

MEYER, M. E. Response inhibition of rats with frontal ablations and caudate-putamen lesions. Psychonomic Science, 1967, 8, 375-376.

ROSVOLD, H. E., \& SZWARCBART, M. K. Neural structures involved in delayed-response performance. In J. M. Warren and K. Akert (Eds.) The frontal granular cortex and behavior. New York: McGraw-Hill Book Co., 1964. Pp. 1-15.

SCHMALTZ, L. W., \& ISAACSON, R. L. Effects of caudate and frontal lesions on retention and relearning of a DRL schedule. Journal of Comparative \& Physiological Psy chology, 1968, 65, 343-348. NOTE

1. Meyer, M. E., and Colby, K. Unpublished study. 